\title{
Evaluation of Risk of Malignancy Index in the Preoperative Assessment of the Patients with Ovarian Mass
}

\author{
Humaira Zafar ${ }^{1}$, Mubashra Naz ${ }^{2}$, Umber Fatima ${ }^{3}$, Anees Fatima ${ }^{4}$, Attiaya Yasmeen ${ }^{5}$
}

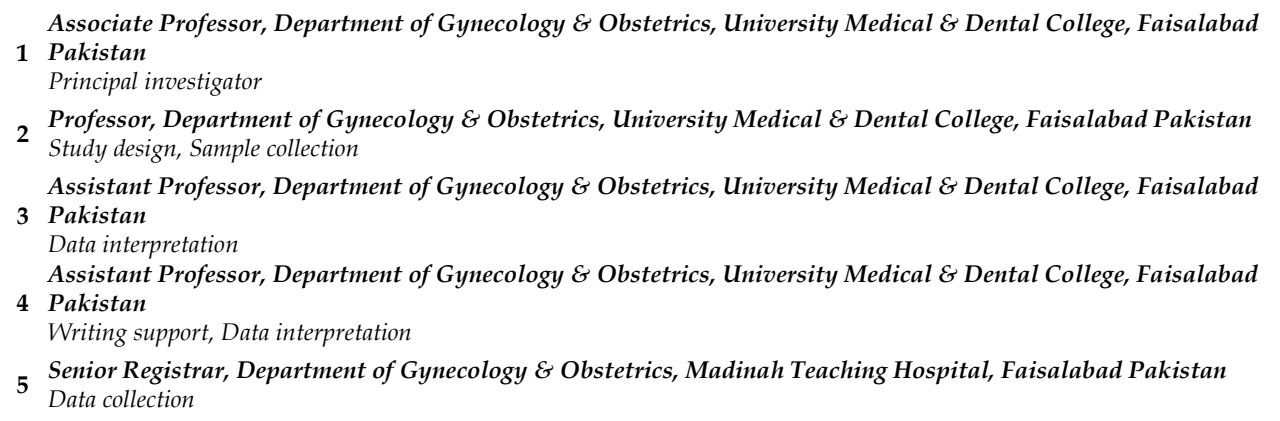

Submitted for Publication: 05-01-2020
Accepted for Publication 28-09-2020
CORRESPONDING AUTHOR
Dr. Humaira Zafar
Associate Professor of Gynecology E Obstetrics,
University Medical E Dental College, Faisalabad
Pakistan
Email: humairazafar75@gmail.com

\section{ABSTRACT}

Background: Adnexal masses indicate a variety of gynecological and non-gynecological disorders, which may be benign or malignant. Early detection of malignancy is crucial to a proper planning of treatment and improvement of survival. Objective: The purpose of the study was to evaluate predictive value of risk of malignancy index (RMI) in differentiating between benign and malignant ovarian masses preoperatively. Study Design: Cross sectional (Validation) study. Settings: Obstetrics \& Gynecology Department of Madinah Teaching Hospital affiliated with University Medical \& Dental College Faisalabad Pakistan. Duration: One year from January 2018 to December 2018. Methodology: Total 86 patients were included in the study. Ultrasound was performed then staging laparotomy done followed by histopathological examination. Results: A total of 86 patients were included in the study. Out of which 50 patients $(58.1 \%)$ were premenopausal and 36 patients $(41.9 \%)$ were menopausal. The mean age of the patients was 55.79 years. According to histopathological examination of the specimens 44 were malignant and 42 were benign. The sensitivity of the RMI for malignant ovarian tumor is $70.45 \%$ and specificity is $69.05 \%$. Conclusion: RMI was considered to have significant importance in preoperative evaluation and treatment of women with an adnexal mass, and was helpful in-patient referral to higher Centre for suitable and effective surgical intervention.

Keywords: RMI, Ovarian malignancy, Adnexal mass.

How to Cite: Zafar H, Naz M, Fatima U, Fatima A, Yasmeen A. Evaluation of Risk of Malignancy Index in the Preoperative Assessment of the Patients with Ovarian Mass. APMC 2020;14(4):299-301. DOI: 10.29054/APMC/2020.835

\section{INTRODUCTION}

Ovarian tumor is the commonest gynecological malignancy in the developing as well as developed countries. Although the mortality rates in patients with ovarian cancer had decreased remarkably over the past years, the incidence is still rising rapidly, even in countries where previously the incidence is low. ${ }^{1}$ It has poor prognosis and most cases (about $80 \%$ ) are at advanced stage at the time of diagnosis. ${ }^{2}$ Precise characterization of adnexal mass is important to decrease anxiety and cost in case of benign tumor. ${ }^{3}$

The purpose of evaluation of ovarian mass is to differentiate between benign and malignant conditions. Early stage diagnosis leads to better planning of treatment and better prognosis. ${ }^{4}$

A variety of prediction tools have been used for evaluation of adnexal mass. ${ }^{5}$ Currently the standard tools for detecting ovarian cancer are abdominopelvic ultrasound, serum CA 125 level and is combined with menopausal status for Risk of Malignancy index (RMI) calculation and is considered simple and affordable test. ${ }^{6}$ The aim of this study is to determine the sensitivity and specificity of RMI so that patients with ovarian pathology may have appropriate management plan preoperatively.

\section{METHODOLOGY}

Study Design: Cross sectional (Validation) study.

Settings: The study was conducted in the Obstetrics \& Gynecology Department of Madinah Teaching Hospital affiliated with University Medical \& Dental College Faisalabad Pakistan.

Duration: One year from January 2018 to December 2018 Sample Technique: Non-probability convenient sampling.

Sample Size: Total number of patients were 87 .

Inclusion Criteria: All patients diagnosed with ovarian tumor admitted in department of Obstetrics and Gynecology were included the study. 
Exclusion Criteria: Females who are diagnosed case of malignant ovarian tumor and on chemotherapy, other adnexal pathology like Tubo-ovarian mass, ectopic pregnancy and pregnant female with ovarian cyst were excluded from the study.

Data Collection Procedure: The approval of the study was taken from the ethical committee of hospital. Informed written consent from all patients was taken.

After detailed history all patients underwent clinical examination. Beside the routine investigations, serum CA 125 level, ultrasound finding and menopausal status of all patients were assessed before surgery.

The RMI 3 was calculated for each woman using the ultrasound score $(\mathrm{U})$, menopausal score $(\mathrm{M})$ and the absolute value of CA 125 by the given method.

RMI = U X M X serum CA125

Five characteristics of ultrasound indicative of malignancy include multilocular, existence of solid components, bilateral lesions., existence of ascites and extra ovarian tumor or presence of metastasis. $U$ is considered 1 if none ore one of these features are found and a score of 3 if two or more of these findings are seen, For menopausal women $\mathrm{M}=3$ and for premenopausal women $M=1$. A value of 200 is taken as cutoff for RMI in this study because it was considered by many studies as the best discriminatory value between benign and malignant ovarian tumor due to its more sensitivity and specificity. Staging Laparotomy of all the patients was performed. The specimen was sent for histopathological examination. The findings of histopathological examination were taken as the gold standard. So, evaluation of MRI done regarding sensitivity, specificity, positive predictive value, negative predictive value and diagnostic accuracy with reference to the histopathological evidence of benign and malignant ovarian tumor. Statistical analysis was done using SPSS version 16.

\section{RESULTS}

Eighty-six patients were included in the study. Out of which 50 patients $(58.1 \%)$ were premenopausal and 36 patients $(41.9 \%)$ were menopausal. (Figure 1)

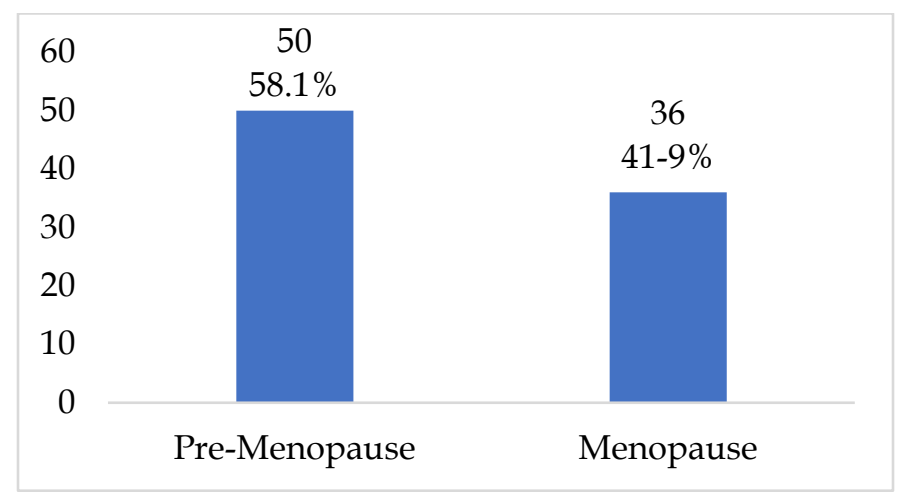

Figure 1: Age ratio in menopause and pre-menopause
The mean age of the patients was 55.79 years (Standard deviation 17.908). According to histopathological examination of the specimens 44 were malignant and 42 were benign.

The sensitivity of the RMI for malignant ovarian tumor is $70.45 \%$ and specificity is $69.05 \%$. (Table 1 )

Table 1: Diagnostic accuracy of risk of malignancy index (RMI)

\begin{tabular}{|c|c|c|c|}
\hline \multirow{2}{*}{ RMI } & \multicolumn{2}{|c|}{ Histopathology } & \multirow{2}{*}{ Total } \\
\cline { 2 - 3 } & Malignant & Benign & \\
\hline$>\mathbf{2 0 0}$ & 31 & 13 & 44 \\
\hline$<\mathbf{2 0 0}$ & 13 & 29 & 42 \\
\hline Total & 44 & 42 & 86 \\
\hline
\end{tabular}

Sensitivity $=70.45 \%$, Specificity $=69.05 \%$, PPV $=70.45 \%$, NPV $=69.05 \%$, Diagnostic Accuracy $=69.77 \%$, Positive likelihood ratio $=2.28$, Negative likelihood ratio $=0.43$

\section{DISCUSSION}

Exploratory laparotomy for evaluation of ovarian pathology had been performed in about $10 \%$ women during their lifetime. ${ }^{7}$ Early diagnosis of malignant ovarian tumor and early referral to a gynecologist result in increased the survival rates of the patients but no single method is available to precisely anticipate ovarian malignancy. ${ }^{8}$ The reliable method of diagnosis are still history, examination and ultrasound findings but has certain limitations so it's possible that gynecologists may find an undiagnosed ovarian malignancy per operatively and resulting in unplanned cytoreduction. If there is a scoring system for prediction of ovarian malignancy, it can help in preoperative counseling, preoperative preparation and patient referral to a specialized Centre if needed. RMI score can be an effective method for prediction of ovarian malignancy, especially in low resource areas. ${ }^{9}$

Most of the studies shows a high diagnostic accuracy at cut- off value of 200. Its usefulness as diagnostic modality depends on the prevalence of ovarian tumor in the study population. ${ }^{10}$ In this study, RMI had a sensitivity of $70.45 \%$ and specificity of $69.05 \%$. Dora SK et al indicated, RMI at a cutoff point of 200 had a sensitivity of $73.9 \%$ and specificity of $96.5 \% .^{8}$ A study conducted in Sweden describe the sensitivity of RMI $89 \%$ and specificity of $80 \% .{ }^{11} \mathrm{Al}$-Asadi JN et al reported that RMI was reliable in preoperative differentiation between malignant and benign adnexal masses at cut off value of 200 with sensitivity and specificity of $100 \%$ and $96.2 \%$ respectively. ${ }^{12}$

Arun-Muthuvel $\mathrm{V}$ et al reported higher sensitivity and specificity than our study $96.1 \%$ and $81 \%$ respectively. ${ }^{13}$ A study conducted in India indicated that RMI $>200$ had a sensitivity of $70.5 \%$ and a specificity of $87.8 \% .{ }^{14}$ 
Although RMI seems to be a reliable method in discrimination between malignant and benign, its utilization in the community depends on the inclination of the clinician to its usage and whether a considerable number of patients with suspected ovarian malignancy will be referred to a gynecologic oncologist with appropriate expertise.

\section{CONCLUSION}

Currently no screening method available for differentiation between benign and malignant ovarian tumor. RMI was considered to have significant importance in preoperative evaluation and treatment of women with an adnexal mass, and was helpful in-patient referral to higher Centre for suitable and effective surgical intervention.

\section{LIMITATIONS}

No limitation felt in conducting study.

\section{SUGGESTIONS / RECOMMENDATIONS}

These studies should be done in different centers, so that data can be shared to identify potentially malignant patient for early intervention with a hope of better outcome of disease.

\section{CONFLICT OF INTEREST / DISCLOSURE}

The authors declare no conflict of interest as regard the publishing of this paper.

\section{ACKNOWLEDGEMENTS}

We are grateful to our teammates providing proficiency that considerably aid the research.

\section{REFERENCES}

1. Qiu L, Yang F, Luo H. A preliminary study: The sequential use of the risk malignancy index and contrast-enhanced ultrasonography in differential diagnosis of adnexal masses. Medicine (Baltimore). 2018;97(29):e11536.
2. Al Musalhi K, Al Kindi M, Al Aisary F, Ramadhan F, Al Rawahi T, Al Hatali K, et al. Evaluation of HE4, CA-125, Risk of Ovarian Malignancy Algorithm (ROMA) and Risk of Malignancy Index (RMI) in the Preoperative Assessment of Patients with Adnexal Mass. Oman Med J. 2016;31(5):336-44.

3. Van Calster B, Valentin L, Van Holsbeke C, Zhang J, Jurkovic D, Lissoni AA, et al. A novel approach to predict the likelihood of specific ovarian tumor pathology based on serum CA-125: a multicenter observational study. Cancer Epidemiol Biomarkers Prev. 2011;20(11):2420-8.

4. Aziz AB, Najmi N. Is Risk Malignancy Index a Useful Tool for Predicting Malignant Ovarian Masses in Developing Countries? Obstet Gynecol Int. 2015;2015:951256.

5. Meys EM, Kaijser J, Kruitwagen RF, Slangen BF, Van Calster B, Aertgeerts $\mathrm{B}$, et al. Subjective assessment versus ultrasound models to diagnose ovarian cancer: A systematic review and metaanalysis. Eur J Cancer. 2016;58:17-29.

6. Al-Musalhi K, Al-Kindi M, Ramadhan F, Al-Rawahi T, Al-Hatali K, Mula-Abed WA. Validity of Cancer Antigen-125 (CA-125) and Risk of Malignancy Index (RMI) in the Diagnosis of Ovarian Cancer. Oman Med J. 2015;30(6):428-34.

7. Baker C, Pasipanodya T, Dwivedi R. The management of suspected ovarian masses in premenopausal women in a DGH setting. BJOG 2013;120(8):372-9.

8. Dora SK, Dandapat AB, Pande B, Hota JP. A prospective study to evaluate the risk malignancy index and its diagnostic implication in patients with suspected ovarian mass. J Ovarian Res. 2017;10(1):55.

9. Davies AP, Jacobs I, Woolas R, Fish A, Oram D. The adnexal mass: benign or malignant? Evaluation of a risk of malignancy index. $\mathrm{Br}$ J Obstet Gynaecol. 1993;100(10):927-31.

10. Simsek HS, Tokmak A, Ozgu E, Doganay M, Danisman N, Erkaya $\mathrm{S}$, et al. Role of a risk of malignancy index in clinical approaches to adnexal masses. Asian Pac J Cancer Prev. 2014;15(18):7793-7.

11. Lycke M, Kristjansdottir B, Sundfeldt K. A multicenter clinical trial validating the performance of HE4, CA125, risk of ovarian malignancy algorithm and risk of malignancy index. Gynecol Oncol. 2018;151(1):159-65.

12. Al-Asadi JN, Al-Maliki SK, Al-Dahhhan F, Al-Naama L, Suood F. The accuracy of risk malignancy index in prediction of malignancy in women with adnexal mass in Basrah, Iraq. Niger J Clin Pract. 2018;21(10):1254-9.

13. Arun-Muthuvel V, Jaya V. Pre-operative evaluation of ovarian tumors by risk of malignancy index, CA125 and ultrasound. Asian Pac J Cancer Prev. 2014;15(6):2929-32.

14. Javdekar R, Maitra N. Risk of Malignancy Index (RMI) in Evaluation of Adnexal Mass. J Obstet Gynaecol India. 2015;65(2):117-21. 